Proceedings

\title{
Analysis of Separability of COVID-19 and Pneumonia in Chest X-ray Images by Means of Convolutional Neural Networks ${ }^{\dagger}$
}

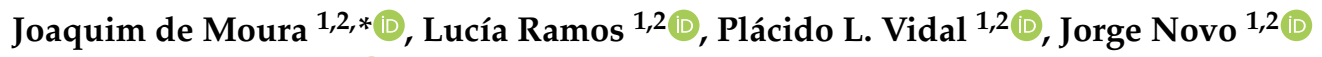 \\ and Marcos Ortega ${ }^{1,2}$ D \\ 1 Centro de investigación CITIC, Universidade da Coruña, 15071 A Coruña, Spain; 1.ramos@udc.es (L.R.); \\ placido.francisco.lizancos.vidal@udc.es (P.L.V.); jnovo@udc.es (J.N.); mortega@udc.es (M.O.) \\ 2 Grupo VARPA, Instituto de Investigación Biomédica de A Coruña (INIBIC), Universidade da Coruña, \\ 15006 A Coruña, Spain \\ * Correspondence: joaquim.demoura@udc.es; Tel.: +34-981-167-000 (ext. 1330) \\ + Presented at the 3rd XoveTIC Conference, A Coruña, Spain, 8-9 October 2020.
}

Published: 21 August 2020

\begin{abstract}
The new coronavirus (COVID-19) is a disease that is caused by severe acute respiratory syndrome coronavirus 2 (SARS-CoV-2). On 11 March 2020, the coronavirus outbreak has been labelled a global pandemic by the World Health Organization. In this context, chest X-ray imaging has become a remarkably powerful tool for the identification of patients with COVID-19 infections at an early stage when clinical symptoms may be unspecific or sparse. In this work, we propose a complete analysis of separability of COVID-19 and pneumonia in chest X-ray images by means of Convolutional Neural Networks. Satisfactory results were obtained that demonstrated the suitability of the proposed system, improving the efficiency of the medical screening process in the healthcare systems.
\end{abstract}

Keywords: computer-aided diagnosis; chest X-ray imaging; COVID-19; pneumonia; deep learning

\section{Introduction}

The coronavirus disease 2019 (COVID-19) disease caused by severe acute respiratory syndrome coronavirus 2 (SARS-CoV-2) has resulted in an unprecedented public health crisis. This highly infectious disease was first identified in the city of Wuhan in Hubei province, China in December 2019. The number of confirmed cases is more than 7.5 million, affecting more than 213 countries, including 287,399 deaths according to the World Health Organization reports. COVID-19 has rapidly progressed to become a global pandemic, causing an unprecedented impact on the health, social, and economic well-being of people around the world. One of the most effective ways of limiting this relevant pandemic disease is the early, rapid, and accurate diagnosis and treatment of infected patients. In this sense, chest $\mathrm{X}$-ray images are the most common and widely available diagnostic imaging technology, playing a crucial role in clinical care and epidemiological studies of confirmed or suspected of COVID-19 cases.

\section{Methodology}

Thus, in this work, we present a novel fully automatic methodology for the analysis of separability of COVID-19 and pneumonia-infected lungs in chest X-ray images, given the high level of similarity between these two lung diseases. To this end, different complementary deep learning-based approaches that are based on a densely convolutional network architecture are adapted to better analyze the distinctive clinical patterns of these relevant diseases [1]. 


\section{Results and Conclusions}

We perform different experiments using two publicly available chest X-ray image datasets in order to validate the designed methodology [2,3]. Satisfactory results were obtained that demonstrated the suitability of the proposed methodology to facilitate early diagnosis and, thus, enable support to the clinical decision-making process in this pandemic scenario. Figure 1 show representative examples of graphical representations of heatmaps based on predictions of pathological regions in the chest X-ray images.
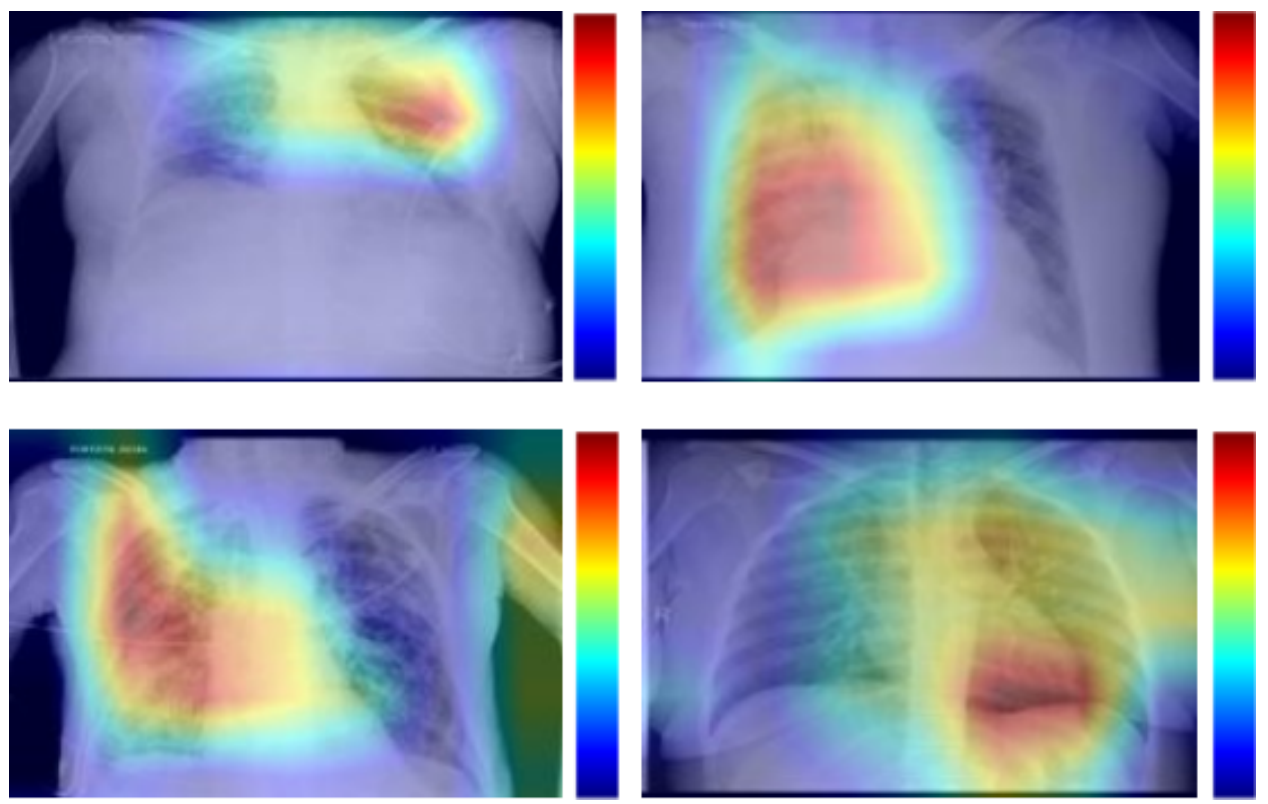

Figure 1. Results of the proposed methodology for the analysis of separability of COVID-19 and pneumonia-infected lungs in chest $X$-ray images. 1st row, chest $X$-ray images from patients infected with pneumonia. 2nd row, chest X-ray images from patients infected with COVID-19.

Author Contributions: J.d.M., L.R. and P.L.V. contributed to the analysis and design of the computer methods and the experimental evaluation methods. J.d.M., J.N. and M.O. contributed with domain-specific knowledge. All the authors performed the result analysis. J.d.M. was in charge of writing the manuscript, and all the authors participated in its critical revision and final approval. All authors have read and agreed to the published version of the manuscript.

Funding: This work is supported by the Instituto de Salud Carlos III, Government of Spain and FEDER funds of the European Union through the DTS18/00136 research projects and by the Ministerio de Ciencia, Innovación y Universidades, Government of Spain through the RTI2018-095894-B-I00 research projects, as well as through Ayudas para la formación de profesorado universitario (FPU), Ref. FPU18/02271. Also, this work has received financial support from the European Union (European Regional Development Fund-ERDF) and the Xunta de Galicia, Centro de Investigación del Sistema Universitario de Galicia, Ref. ED431G 2019/01.

Conflicts of Interest: The authors declare no conflict of interest.

\section{References}

1. De Moura, J.; Ramos, L.; Vidal, P.L.; Cruz, M.; Abelairas, L.; Castro, E.; Ortega, M. Fully automatic deep convolutional approaches for the analysis of covid-19 using chest X-ray images. medRxiv 2020, 1-13. 
2. Pneumonia Detection Challenge, Radiological Society of North America (RSNA). Available online: https: / / www.kaggle.com/c/rsna-pneumonia-detection-challenge/ (accessed on 10 April 2020)

3. COVID-19 DATABASE, Italian Society of Medical Radiology (SIRM). Available online: https://www.sirm. org/category/senza-categoria/covid-19/ (accessed on 10 April 2020)

(C) 2020 by the authors. Licensee MDPI, Basel, Switzerland. This article is an open access article distributed under the terms and conditions of the Creative Commons Attribution (CC BY) license (http:/ / creativecommons.org/licenses/by/4.0/). 